NSF-ITP-00-122

\title{
Finite temperature resolution of the Klebanov-Tseytlin singularity
}

\author{
Alex Buchelf \\ Institute for Theoretical Physics \\ University of California \\ Santa Barbara, CA 93106-4030, U.S.A.
}

\begin{abstract}
Naked singularities in the gravitational backgrounds dual to gauge theories can be hidden behind the black hole horizon. We present an exact black hole solution in the Klebanov-Tseytlin geometry [hepth/0002159. Our solution realizes Maldacena dual of the finite temperature $\mathcal{N}=1$ duality cascade of [hep-th/0007191] above the temperature of the chiral symmetry breaking. We compare the BekensteinHawking entropy of the black hole with the entropy of the $S U(N+$ $M) \times S U(N)$ gauge theory.
\end{abstract}

NOTE ADDED: The non-extremal generalization of the KT background proposed in this paper was obtained partly with numerical methods. In hep-th/0102105 this solution has been rederived analytically, and a numerical error pointed that led to the conclusion of a non-singular horizon in the non-BPS background discussed here. In hep-th/0102105] it is shown that within the self-dual ansatz for the three form fluxes the unique non-extremal solution (discussed in this paper) always has singular horizon for any value of non-extremality. Thus, the constant dilaton ansatz (following from the self-duality of the three form fluxes) appears to be too restrictive to describe the high temperature phase of the cascading gauge theory. The system of second order equations in the radial variable describing non-extremal

\footnotetext{
${ }^{1}$ buchel@itp.ucsb.edu
} 
generalizations of the KT background whose solutions may have regular horizons is given in hep-th/0102105. (Added) section 5 of this paper discusses numerical error of the previous version, identified in hep-th/0102105. 


\section{Introduction}

The AdS/CFT duality of Maldacena [1] relating supergravity and strongly coupled superconformal gauge theories is usually motivated by comparing stacks of elementary branes with corresponding gravitational backgrounds in string or M-theory. For example, a large number $N$ of coincident D3branes and the 3-brane classical solution leads to the duality between $\mathcal{N}=4$ supersymmetric gauge theory and Type IIB strings on $A d S_{5} \times S^{5}$ [近, 2, 包.

Subsequently, the duality has been extended to nonconformal systems and to systems with less supersymmetry 团. One way to break supersymmetry of the world-volume theory of the D3-branes is to place them at appropriate conical singularities [5, 6, 7, 8]. Then the background dual to the CFT on the D3-branes is $A d S_{5} \times X_{5}$ where $X_{5}$ is the Einstein manifold which is the base of the cone. Breaking conformal invariance without further breaking the supersymmetry can be achieved by placing fractional D3 branes at the singularity in addition to the regular ones. These fractional D3 branes are D5-branes wrapped over (collapsed) 2-cycle at the singularity [9]. In the case of a conifold the singularity is a point. Placing $M$ fractional and $N$ regular D3-branes Klebanov and Nekrasov constructed [10] the renormalization group flow in the gravity dual to the $\mathcal{N}=1$ supersymmetric $S U(N) \times S U(N+M)$ gauge theory]. The supergravity equations were solved to leading order in $M / N$ in [10]. In [12], this solution (which we refer to from now on as KT) was completed to all orders; the conifold suffers logarithmic warping, and the relative gauge coupling of the two gauge factors runs logarithmically at all scales. The D3-brane charge, i.e the 5-form flux, decreases logarithmically as well. However, the logarithm in the solution is not cut off at small radius; eventually the D3-charge becomes negative and one encounters a naked singularity in the metric.

The appearance of naked singularities in the gravitational dual of the nonconformal gauge theories with reduced supersymmetry is rather common

\footnotetext{
${ }^{1}$ Renormalization group flows on fractional D3-branes at an orbifolded conifold were discussed in [11].
} 
phenomenon. Thus, understanding the physics of these singularities is an important problem. By now we know several different mechanisms for resolving singularities, which seem to depend on the amount of supersymmetry present in the problem. In the case when the gauge theory has 8 supercharges $(\mathcal{N}=2$ supersymmetry in $D=4)$ the naked singularity, known as repulson, is resolved by the enhancon mechanism characterized by the expansion of a system of branes in the transverse directions [13, 14, 15, 16, 17, 18. In the class of $\mathcal{N}=1$ gauge theories obtained by mass deformation of parent $\mathcal{N}=4$ gauge theories, the naked singularity of the gravitational dual is resolved by Dp-brane polarization into a spherical Dp+2-brane [19, 20, 21, 22, 23, 24 via Myer's dielectric effect [25]. A common theme in the enhancon and the brane polarization mechanism is that the singularity of the gravitation background is resolved by a distinctive string/braney phenomena. The resolution of the singularity in the KT geometry is rather different. Klebanov and Strassler 26] showed that in this case the resolution is achieved entirely within supergravity by deforming the conifold. The crucial observation made in [26] was the identification of the 5 -form flux decrease in KT with the repeated chain of Seiberg-duality transformations in which $N \rightarrow N-M$. At the IR end of the duality cascade the gauge theory confines with the chiral symmetry breaking. Klebanov and Strassler convincingly argued that the chiral symmetry breaking on the gauge theory side is dual to deforming the conifold on the gravity side of the Maldacena duality.

The resolutions of singularities we mentioned above, are for the gravitational backgrounds dual to the gauge theories at zero temperature. There is an extensive literaturef on the application of gravity/gauge theory duality to the finite temperature gauge theories starting with the work of Witten [27]. Finite temperature gauge theories have black hole gravitational dual. Thus, one can imagine that by "heating" the gauge theory, the naked singularity of its gravitational dual will be hidden behind the black hole horizon. In fact, this underlines the classification of naked singularities proposed in 228 ]: the

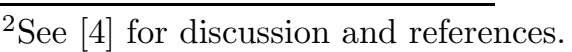

${ }^{3}$ In 28] this criterion has been applied for admissibility of singular solutions with four
} 
"good" naked singularities are those which can be obtained as limits of regular black holes. One of the motivations for the criterion [28] is that finite temperature in field theory serves as an infrared cutoff in the sense that it masks physics at scales lower than the temperature. A naked singularity that can be hidden behind a black hole horizon is a signal of a non-trivial but sensible infrared physics in the dual gauge theory. In particular, this might signal of a phase transition in the gauge theory. If we approach a "good" naked singularity by taking a limit of regular black holes, the dual picture is that the infrared cutoff (finite temperature) is being removed. As the temperature is sufficiently lowered, the horizon should retreat until the original singular geometry is recovered. For the KT singularity we can turn the argument around: since we know that KT singularity is "good" - we know of its resolution - there must exist a black hole solution with the asymptotic KT geometry. Another way to reach the same conclusion is to recall that at zero temperature, the naked singularity of the gravitational dual was resolved by the mechanism dual to the chiral symmetry breaking in the gauge theory. However, at high enough temperature, we expect restoration of the chiral symmetry in the gauge theory. Thus, the Maldacena dual of this gauge theory at temperature above chiral symmetry breaking should be described by a black hole in KT geometryf. In this paper we construct such a black hole solution.

A finite temperature generalization of Polchinski-Strassler construction [19] has been recently discussed in [29]. It was also emphasized there that in the high temperature phase it is unnecessary to invoke Myers' mechanism since there are no naked singularities. The gravity solution was found in 29] in the leading order in mass perturbation.

This paper is organized as follows. We start the next section by introducing our conventions and recalling $\mathrm{KT}$ solution. We then describe an exact black hole solution in KT geometry. In section 3 we compute the Bekenstein-

dimensional Poincare invariance in five-dimensional gravity with scalars.

${ }^{4}$ Note that the chiral symmetry breaking phase transition is precisely the example of a non-trivial infrared dynamics which can be hidden by the IR cutoff. 
Hawking entropy of this black hole and compare it to the entropy of the dual gauge theory. We conclude in section 4 .

\section{Black hole solution in KT geometry}

\subsection{Type IIB equations of motion}

We use mostly negative conventions for the signature $(+-\cdots-)$ and $\epsilon^{1 \cdots 10}=$ +1 . The type IIB equations consist of [30]:

- The Einstein equations:

$$
R_{M N}=T_{M N}^{(1)}+T_{M N}^{(3)}+T_{M N}^{(5)}
$$

where the energy momentum tensors of the dilaton/axion field, $B$, the three index antisymmetric tensor field, $F_{(3)}$, and the self-dual five-index tensor field, $F_{(5)}$, are given by

$$
\begin{gathered}
T_{M N}^{(1)}=P_{M} P_{N}{ }^{*}+P_{N} P_{M}{ }^{*} \\
T_{M N}^{(3)}=\frac{1}{8}\left(G^{P Q}{ }_{M} G_{P Q N}^{*}+G^{* P Q}{ }_{M} G_{P Q N}-\frac{1}{6} g_{M N} G^{P Q R} G_{P Q R}^{*}\right), \\
T_{M N}^{(5)}=\frac{1}{6} F^{P Q R S}{ }_{M} F_{P Q R S N} .
\end{gathered}
$$

In the unitary gauge $B$ is a complex scalar field and

$$
P_{M}=f^{2} \partial_{M} B, \quad Q_{M}=f^{2} \operatorname{Im}\left(B \partial_{M} B^{*}\right),
$$

with

$$
f=\frac{1}{\left(1-B B^{*}\right)^{1 / 2}},
$$

while the antisymmetric tensor field $G_{(3)}$ is given by

$$
G_{(3)}=f\left(F_{(3)}-B F_{(3)}^{*}\right)
$$

- The Maxwell equations:

$$
\left(\nabla^{P}-i Q^{P}\right) G_{M N P}=P^{P} G_{M N P}^{*}-\frac{2}{3} i F_{M N P Q R} G^{P Q R} .
$$


- The dilaton equation:

$$
\left(\nabla^{M}-2 i Q^{M}\right) P_{M}=-\frac{1}{24} G^{P Q R} G_{P Q R} .
$$

- The self-dual equation:

$$
F_{(5)}=\star F_{(5)} .
$$

In addition, $F_{(3)}$ and $F_{(5)}$ satisfy Bianchi identities which follow from the definition of those field strengths in terms of their potentials:

$$
\begin{aligned}
& F_{(3)}=d A_{(2)}, \\
& F_{(5)}=d A_{(4)}-\frac{1}{8} \operatorname{Im}\left(A_{(2)} \wedge F_{(3)}^{*}\right) .
\end{aligned}
$$

We consider the background with constant dilaton and zero axion

$$
B=\frac{1-g_{s}}{1+g_{s}} .
$$

We also use

$$
G_{3}=f(1-B) \mathcal{F}_{3}+i f(1+B) H_{3},
$$

where $\mathcal{F}_{3}$ and $H_{3}$ are the RR and NSNS 3-form field strength correspondingly.

\section{$2.2 \quad$ KT solution}

In this section we review KT 12 gravitational dual of the $S U(N) \times S U(N+$ $M$ ) gauge theory, mainly to set up our notations, and discuss the dictionary between two descriptions.

The Einstein frame metric is:

$$
d s_{10}^{2}=c_{1}(\tau)^{2}\left[d t^{2}-d \bar{x}^{2}\right]-c_{2}(\tau)^{2}\left[d \tau^{2}+9 d s_{T^{1,1}}^{2}\right],
$$

where $d s_{T^{1,1}}^{2}$ is the metric on the coset space $T^{1,1}=(S U(2) \times S U(2)) / U(1)$ which is the base of a cone 31]

$$
d s_{T^{1,1}}^{2}=\frac{1}{9}\left(g^{5}\right)^{2}+\frac{1}{6} \sum_{i=1}^{4}\left(g^{i}\right)^{2} .
$$


We use the following basis of 1-forms on the compact space [32]

$$
\begin{aligned}
& g^{1}=\left(-\sin \theta_{1} d \phi_{1}-\cos \psi \sin \theta_{2} d \phi_{2}+\sin \psi d \theta_{2}\right) / \sqrt{2}, \\
& g^{2}=\left(d \theta_{1}-\sin \psi \sin \theta_{2} d \phi_{2}-\cos \psi d \theta_{2}\right) / \sqrt{2}, \\
& g^{3}=\left(-\sin \theta_{1} d \phi_{1}+\cos \psi \sin \theta_{2} d \phi_{2}-\sin \psi d \theta_{2}\right) / \sqrt{2}, \\
& g^{4}=\left(d \theta_{1}+\sin \psi \sin \theta_{2} d \phi_{2}+\cos \psi d \theta_{2}\right) / \sqrt{2}, \\
& g^{5}=d \psi+\cos \theta_{1} d \phi_{1}+\cos \theta_{2} d \phi_{2} .
\end{aligned}
$$

Furthermore,

$$
\begin{aligned}
& c_{1}(\tau)=H(\tau)^{-1 / 4}, \\
& c_{2}(\tau)=\epsilon H(\tau)^{1 / 4} \frac{e^{\tau / 3}}{3}, \\
& H(\tau)=b_{0}+\frac{81}{2} g_{s} M^{2} e^{-4 \tau / 3}\left[\frac{\tau}{3}-\ln \frac{r_{s}}{\epsilon}\right] .
\end{aligned}
$$

Introducing a new radial variable

$$
r=\epsilon e^{\tau / 3},
$$

and eliminating asymptotically flat region (setting $b_{0}=0$ ) we can rewrite (2.14) as

$$
d s_{10}^{2}=H(r)^{-1 / 2}\left[d t^{2}-d \bar{x}^{2}\right]-H(r)^{1 / 2}\left[d r^{2}+r^{2} d s_{T^{1,1}}^{2}\right],
$$

with

$$
H(r)=\frac{L^{4}}{r^{4}} \ln \frac{r}{r_{s}}, \quad L^{4}=\frac{81}{2} g_{s} M^{2} \epsilon^{4} .
$$

As we already mentioned, there is a naked singularity in KT geometry. From (2.20) we see that it is at $r=r_{s}$.

The dilaton is constant, and the three-form field strength

$$
G_{3}=\frac{1}{2} g_{s}^{1 / 2} M\left[g_{3} \wedge g_{4} \wedge g_{5}+g_{1} \wedge g_{2} \wedge g_{5}-i d(\tau) \wedge g_{3} \wedge g_{4}-i d(\tau) \wedge g_{1} \wedge g_{2}\right] .
$$

is self-dual

$$
G_{3}=i \star_{6} G_{3}
$$


Finally, the five-form field strength is

$$
F_{5}=\mathcal{F}_{5}+\star \mathcal{F}_{5}
$$

with

$$
\mathcal{F}_{5}=\frac{1}{4}\left[c_{1}(\tau)^{4}\right]^{\prime} d t \wedge d x_{1} \wedge d x_{2} \wedge d x_{3} \wedge d(\tau) .
$$

In (2.24) (and below) the prime denotes the derivative with respect to $\tau$.

The dictionary between gauge and gravity descriptions [10, 12, 26] is most clear when one uses $r(2.18)$ as a radial coordinate on the gravity side; $\mu \equiv r / \epsilon^{2}$ is then natural to identify with the RG scale of the gauge theory. On the gauge theory side, at a given energy scale $\mu$, we have $S U(N+M) \times$ $S U(N)$ gauge theory with two chiral superfields $A_{1}, A_{2}$ in the $(N+M, \bar{N})$ representation, and two fields $B_{1}, B_{2}$ in the $(\overline{N+M}, N)$ representation. The superpotential of the model is

$$
W \sim \operatorname{tr}\left(A_{i} B_{j} A_{k} B_{\ell}\right) \epsilon^{i k} \epsilon^{j \ell}
$$

The $S U(2) \times S U(2) \times U(1)$ global symmetry of the $T^{1,1}$ is realized as a global symmetry of the gauge theory with the first (second) $S U(2)$ factor rotating the flavor index of the $A_{i}\left(B_{i}\right)$, while the "baryon" $U(1)$ acts as $A_{i} \rightarrow A_{i} e^{i \alpha}$, $B_{i} \rightarrow B_{i} e^{-i \alpha}$. The two gauge group factors have gauge couplings $g_{1}$ and $g_{2}$. Under the RG flow, the sum of the couplings does not run. It is dual to the dilaton of in $\mathrm{KT}$ background:

$$
\frac{1}{g_{s}}=\frac{4 \pi}{g_{1}^{2}}+\frac{4 \pi}{g_{2}^{2}} .
$$

On the gauge theory side, the difference between two couplings is

$$
\frac{4 \pi}{g_{2}^{2}}-\frac{4 \pi}{g_{1}^{2}} \sim M \ln (\mu / \Lambda)[3+2(1-\gamma)],
$$

where $\gamma$ is the anomalous dimension of operators $\operatorname{tr} A_{i} B_{j}$. This is dual on the gravity side to the NSNS 2-form flux $B_{2}$ through the $S^{2}$ of the cone base:

\footnotetext{
${ }^{5}$ Topologically $T^{1,1}$ is $S^{2} \times S^{3}$.
}

$$
\frac{1}{g_{s}} \int_{S^{2}} B_{2} \sim M \ln (r / \epsilon) \sim M \ln (\mu / \Lambda),
$$


where we identified $\Lambda \sim 1 / \epsilon$. It is clear from (2.26), (2.27) that starting at some energy scale and flowing either to the UV or the IR one inevitably hits a Landau pole - one of the two gauge couplings will become infinitely large. Klebanov and Strassler argued that this singularity in the gauge theory is artificial, and arises as one insists on describing strongly coupled gauge dynamics through the perturbative degrees of freedom. Rather, [26] one has to do a Seiberg duality [33] on the strongly coupled gauge factor, which becomes weakly coupled in terms of a dual, "magnetic" description. We will not discuss the details here; only collect the necessary facts. Turns out that the Seiberg duality transformation is a self-similarity transformation of the gauge theory which replaces $N \rightarrow N-M$ as one flows to the IR, or $N \rightarrow N+M$ as the energy increases. Thus, effectively, the rank of the gauge group is not constant along the RG flow, but changes with energy:

$$
N=N(\mu) \sim M^{2} \ln (\mu / \Lambda) .
$$

(To see (2.29) note that the rank changes by $\triangle N \sim M$ as $M \triangle(\ln (\mu / \Lambda)) \sim 1$.) This fits nicely with the result of the gravitational dual where the five-form field strength with components along the $T^{1,1}$ grows as

$$
F_{5}=\mathcal{F}_{5}+\star \mathcal{F}_{5}, \quad \star \mathcal{F}_{5} \sim g_{s} M^{2} \ln (r / \epsilon) \operatorname{vol}\left(T^{1,1}\right) .
$$

The original Seiberg duality [33] was proposed between two different gauge theories in the UV which flow in the IR to the same superconformal fixed point. The duality cascade of Klebanov-Strassler is an extension of the

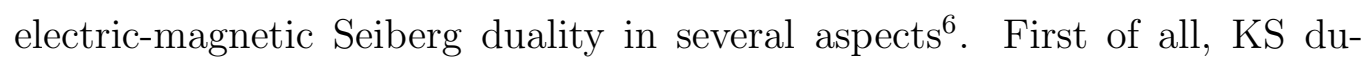
ality is an equivalence between nonconformal theories. Here, there is no anomaly-free $U(1)_{R}$ symmetry which in the case of Seiberg duality was essential to match chiral rings of two theories. From the purely field theoretic perspective, one also can not use chiral anomalies of global symmetries to argue for the equivalence of two dual descriptions - the only unbroken global symmetries of the problem are $S U(2) \times S U(2) \times U(1)$ symmetry of the conifold: $S U(2)$ symmetries don't have chiral anomalies and the "baryon" $U(1)$

\footnotetext{
${ }^{6}$ I would like to thank Matt Strassler for discussing this point.
} 
is not chiral. Second, unlike the original Seiberg duality which is true in the far IR, dualities of KS arise at finite energies. Third, in [26] duality has been performed on only one gauge factor while the other one was a spectator. Strictly speaking, to argue for the duality the coupling of that gauge factor has to be exactly zero. However, KS duality changes couplings of both gauge factors, thus the latter assumption seems inconsistent.

Above arguments indicate that conventional tools used to check dualities of $\mathcal{N}=1$ gauge theories are not very useful for KS dualities. The strongest support for KS duality cascade comes from the behavior of the five-form filed strength of its gravitational dual. In section 3 of this paper we present another evidence in favor of KS duality cascade: we show that the BekensteinHawking entropy of a black hole in KT geometry matches (up to numerical coefficients) the entropy of the $S U(N(\mu)) \times S U(N(\mu)+M)$ gauge theory.

\subsection{Black hole solution in KT geometry}

Black holes have long been objects of interests in string theory ${ }^{7}$. In the context of AdS/CFT duality, they realize gravitational dual of gauge theories at finite temperature. In this section we present an exact solution of type IIB equations of motion describing a black hole in KT geometry. The solution realizes the gravitational dual of KS cascade of dualities [26] at temperatures above chiral symmetry breaking; it is also the solution which can "hide" naked singularity of KT geometry.

We start with the following ansatz for the metric

$$
d s_{10}^{2}=c_{1}(\tau)^{2}\left[\triangle_{1}(\tau)^{2} d t^{2}-d \bar{x}^{2}\right]-c_{2}(\tau)^{2}\left[\triangle_{2}(\tau)^{-2} d \tau^{2}+9 d s_{T^{1,1}}^{2}\right],
$$

where we introduce two new warp factor $\triangle_{1}(\tau)$ and $\triangle_{2}(\tau)$, such that $\triangle_{i}(\tau) \rightarrow$ 1 as $\tau \rightarrow \infty$. Also we expect to find zeros of the warp factors at finite $\tau$ s.

We will look for the solution with self-dual three form field strength (2.22); this insures that the dilaton is constant. The RR 3-form ansatz we take is

$$
\mathcal{F}_{3}=M g_{5} \wedge g_{3} \wedge g_{4}+d\left[f_{1}(\tau) g_{1} \wedge g_{2}+f_{2}(\tau) g_{1} \wedge g_{3}+f_{3}(\tau) g_{1} \wedge g_{4}\right.
$$

\footnotetext{
${ }^{7}$ For an introduction to the subject and references see [34].
} 


$$
\begin{aligned}
& +f_{4}(\tau) g_{1} \wedge g_{5}+f_{5}(\tau) g_{2} \wedge g_{3}+f_{6}(\tau) g_{2} \wedge g_{4}+f_{7}(\tau) g_{2} \wedge g_{5} \\
& \left.+f_{8}(\tau) g_{3} \wedge g_{4}+f_{9}(\tau) g_{3} \wedge g_{5}+f_{10}(\tau) g_{4} \wedge g_{5}\right]
\end{aligned}
$$

while the one for the NSNS 3-form

$$
\begin{aligned}
H_{3}= & d\left[h_{1}(\tau) g_{1} \wedge g_{2}+h_{2}(\tau) g_{1} \wedge g_{3}+h_{3}(\tau) g_{1} \wedge g_{4}\right. \\
& +h_{4}(\tau) g_{1} \wedge g_{5}+h_{5}(\tau) g_{2} \wedge g_{3}+h_{6}(\tau) g_{2} \wedge g_{4}+h_{7}(\tau) g_{2} \wedge g_{5} \\
& \left.+h_{8}(\tau) g_{3} \wedge g_{4}+h_{9}(\tau) g_{3} \wedge g_{5}+h_{10}(\tau) g_{4} \wedge g_{5}\right] .
\end{aligned}
$$

In (2.32) $M$ is a number. $g_{5} \wedge g_{3} \wedge g_{4}$ is a closed 3-form on $T^{1,1}$ which is not exact. Clearly,

$$
d G_{3}=0 .
$$

Furthermore, we use the black p-brane ansatz for the 5-form:

$$
\begin{aligned}
& F_{5}=\mathcal{F}_{5}+\star \mathcal{F}_{5} \\
& \mathcal{F}_{5}=\frac{\omega(\tau) c_{1}(\tau)^{4} c_{2}(\tau) \triangle_{1}(\tau)}{\triangle_{2}(\tau)} d t \wedge d x_{1} \wedge d x_{2} \wedge d x_{3} \wedge d(\tau)
\end{aligned}
$$

With our ansatz for the 3-forms, the self-duality (2.22) constraints

$$
\begin{aligned}
h_{5}(\tau) & =-h_{3}(\tau), \\
f_{5}(\tau) & =-f_{3}(\tau), \\
f_{6}(\tau) & =f_{2}(\tau) \\
h_{6}(\tau) & =h_{2}(\tau), \\
f_{4}(\tau) & =0 \\
h_{4}(\tau) & =0 \\
f_{9}(\tau) & =0 \\
h_{9}(\tau) & =0 \\
f_{10}(\tau) & =f_{7}(\tau)=0, \\
h_{10}(\tau) & =h_{7}(\tau)=0, \\
f_{3}^{\prime}(\tau) & =0 \\
h_{3}^{\prime}(\tau) & =0
\end{aligned}
$$




$$
\begin{aligned}
f_{8}^{\prime}(\tau) & =\frac{h_{2}(\tau)}{g_{s} \triangle_{2}(\tau)}, \\
h_{8}^{\prime}(\tau) & =-\frac{g_{s} f_{2}(\tau)}{\triangle_{2}(\tau)} \\
f_{2}^{\prime}(\tau) & =\frac{1}{2 g_{s} \triangle_{2}(\tau)}\left(h_{1}(\tau)-h_{8}(\tau)\right), \\
h_{2}^{\prime}(\tau) & =\frac{g_{s}}{2 \triangle_{2}(\tau)}\left(f_{8}(\tau)-f_{1}(\tau)\right), \\
f_{1}^{\prime}(\tau) & =-\frac{h_{2}(\tau)}{g_{s} \triangle_{2}(\tau)}, \\
h_{1}^{\prime}(\tau) & =\frac{g_{s}}{\triangle_{2}(\tau)}\left(f_{2}(\tau)-M\right) .
\end{aligned}
$$

In KT solution (from (2.21))

$$
\begin{aligned}
\triangle_{1}(\tau) & =\triangle_{2}(\tau)=1 \\
f_{1}(\tau) & =f_{8}(\tau)=h_{2}(\tau)=0, \\
f_{2}(\tau) & =\frac{1}{2} M \\
h_{1}(\tau) & =h_{8}(\tau)=-\frac{1}{2} g_{s} M \tau .
\end{aligned}
$$

We would like to have asymptotically as $\tau \rightarrow \infty$ KT solution, so we choose the following solution of (2.36)

$$
\begin{aligned}
f_{1}(\tau) & =f_{8}(\tau)=h_{2}(\tau)=0, \\
f_{2}(\tau) & =\frac{1}{2} M, \\
h_{8}(\tau) & =h_{1}(\tau) .
\end{aligned}
$$

All constraints of $(2.36)$ are reduced to a single ODE

$$
h_{1}^{\prime}(\tau)=-\frac{g_{s} M}{2 \triangle_{2}(\tau)}
$$

The 3-form Maxwell equations are satisfied provided

$$
\omega(\tau)=\frac{1}{4} \frac{\triangle_{2}(\tau)}{c_{1}(\tau)^{4} c_{2}(\tau) \triangle_{1}(\tau)}\left[\triangle_{1}(\tau) c_{1}(\tau)^{4}\right]^{\prime}
$$


Consider now the Einstein equations (2.1). The dilaton/axion is constant in our background, so

$$
R_{M N}=T_{M N}^{(3)}+T_{M N}^{(5)} .
$$

The energy-momentum tensor of the five-form is diagonal traceless

$$
T_{M N}^{(5)}=4 \omega(\tau)^{2} \operatorname{diag}\{1,-1,-1,-1,-1,1,1,1,1,1\} .
$$

The energy-momentum tensor of the three-form is diagonal as well

$$
T_{M N}^{(3)}=\frac{g_{s} M^{2}}{18 c_{2}(\tau)^{6}} \operatorname{diag}\{1,-1,-1,-1,1,1,1,1,1,1\},
$$

where we used constraints (2.38) and (2.39). From (2.41) it now follows that

$$
R_{11}+R_{22}=0,
$$

which gives a differential equation relating warp factors $\triangle_{i}(\tau)$ :

$$
\begin{aligned}
R_{11}+R_{22}= & 0 \\
= & \frac{\triangle_{2}(\tau)}{c_{1}(\tau) c_{2}(\tau)^{3} \triangle_{1}(\tau)}\left[4 \triangle_{2}(\tau)\left[\triangle_{1}(\tau)\right]^{\prime}\left[c_{1}(\tau) c_{2}(\tau)\right]^{\prime}\right. \\
& \left.+c_{1}(\tau) c_{2}(\tau)\left[\triangle_{2}(\tau)\left[\triangle_{1}(\tau)\right]^{\prime}\right]^{\prime}\right]
\end{aligned}
$$

The latter equation is easy to integrate once:

$$
\triangle_{2}(\tau)\left[\triangle_{1}(\tau)\right]^{\prime}=\frac{A}{c_{1}(\tau)^{4} c_{2}(\tau)^{4}} \equiv h(\tau),
$$

where $A$ is an integration constant. For later convenience we introduce $h(\tau)$ as in (2.46). Note that for the $c_{i}(\tau)$ in the original KT solution

$$
h(\tau)=\frac{81 A}{\epsilon^{4}} e^{-4 \tau / 3},
$$

and $h(\tau) \rightarrow 0$ as $\tau \rightarrow \infty$. This fact will be important later, as it will turn out that we can use the same $h(\tau)$ here.

At this stage it is convenient to identify constraint from the five-form Bianchi identity. Using (2.46), we find:

$$
\left[c_{1}(\tau)\right]^{\prime \prime}=\frac{1}{36 \triangle_{1}(\tau)^{2} \triangle_{2}(\tau)^{2} c_{1}(\tau)^{7} c_{2}(\tau)^{8}}(
$$




$$
\begin{aligned}
& 9 A^{2}+2 g_{s} M^{2} \triangle_{1}(\tau)^{2} c_{1}(\tau)^{8} c_{2}(\tau)^{4} \\
& +36 \triangle_{1}(\tau) \triangle_{2}(\tau) A c_{1}(\tau)^{3} c_{2}(\tau)^{4}\left[c_{1}(\tau)\right]^{\prime} \\
& +36 \triangle_{1}(\tau)^{2} \triangle_{2}(\tau)^{2} c_{1}(\tau)^{6} c_{2}(\tau)^{8}\left(\left[c_{1}(\tau)\right]^{\prime}\right)^{2} \\
& -144 \triangle_{1}(\tau)^{2} \triangle_{2}(\tau)^{2} c_{1}(\tau)^{7} c_{2}(\tau)^{7}\left[c_{1}(\tau)\right]^{\prime}\left[c_{2}(\tau)\right]^{\prime} \\
& \left.-36 \triangle_{1}(\tau)^{2} \triangle_{2}(\tau) c_{1}(\tau)^{7} c_{2}(\tau)^{8}\left[\triangle_{2}(\tau)\right]^{\prime}\left[c_{1}(\tau)\right]^{\prime}\right)
\end{aligned}
$$

Now we are left with three independent Einstein equations (2.41) for the components $M=N=\{1,5,6\}$. Turns out, that given (2.40), 2.46) and (2.48), the $M=N=1$ Einstein equation is satisfied automatically, while the other two give a nonlinear system of ordinary differential equations on $h(\tau)$ and $\triangle_{1}(\tau)$ :

$$
\begin{aligned}
0= & 2 h(\tau)\left(\left[\triangle_{1}(\tau)\right]^{\prime}\right)^{3}-5\left(\triangle_{1}(\tau)\right)^{2}[h(\tau)]^{\prime}\left[\triangle_{1}(\tau)\right]^{\prime \prime} \\
& -5 \triangle_{1}(\tau)[h(\tau)]^{\prime}\left(\left[\triangle_{1}(\tau)\right]^{\prime}\right)^{2}+5 \triangle_{1}(\tau)^{2}\left[\triangle_{1}(\tau)\right]^{\prime}[h(\tau)]^{\prime \prime} \\
0= & 9 \triangle_{1}(\tau)\left[\triangle_{1}(\tau)\right]^{\prime}\left([h(\tau)]^{\prime}\right)^{2}+9 \triangle_{1}(\tau) h(\tau)[h(\tau)]^{\prime}\left[\triangle_{1}(\tau)\right]^{\prime \prime} \\
& -9 h(\tau)[h(\tau)]^{\prime}\left(\left[\triangle_{1}(\tau)\right]^{\prime}\right)^{2}-9 \triangle_{1}(\tau) h(\tau)\left[\triangle_{1}(\tau)\right]^{\prime}[h(\tau)]^{\prime \prime} \\
& -16 \triangle_{1}(\tau)\left(\left[\triangle_{1}(\tau)\right]^{\prime}\right)^{3}
\end{aligned}
$$

for $M=N=5$ and $M=N=6$ correspondingly. A trick to solve (2.49) is to notice that $\triangle_{1}(\tau)$ has only implicit $\tau$ dependence through $h(\tau)$, that is,

$$
\triangle_{1}(\tau)=\triangle_{1}(h(\tau))
$$

This should not come as a surprise since from (2.46) it is clear that by changing a radial coordinate $\tau$, i.e. redefining $\tau \rightarrow \tilde{\tau}=\tilde{\tau}(\tau)$, one changes $h(\tau) \rightarrow \tilde{h}(\tau)=h(\tau) d \tau / d \tilde{\tau}$. One obvious solution (which eventually gives original KT solution) is then $h(\tau)=0$. As we are interested in the black hole solutions which have nontrivial warp factors, we assume $[h(\tau)]^{\prime} \neq 0$. Eqs. (2.49) are then reduced to two ordinary differential equations on a single function

$$
f(h) \equiv\left(\triangle_{1}(h(\tau))\right)^{2},
$$


namely,

$$
\begin{aligned}
0= & x\left([f(x)]^{\prime}\right)^{3}-10 f(x)^{2}[f(x)]^{\prime \prime} \\
0= & 9 f(x)[f(x)]^{\prime}-9 x\left([f(x)]^{\prime}\right)^{2} \\
& +9 x f(x)[f(x)]^{\prime \prime}-4\left([f(x)]^{\prime}\right)^{3},
\end{aligned}
$$

where the prime denotes the derivative with respect to $x$. Turns out, both equations are solved simultaneously provided

$$
[f(x)]^{\prime}=\frac{3}{2} \frac{\left(30 x \pm 2 \sqrt{135 x^{2}+400 f(x)}\right) f(x)}{9 x^{2}-40 f(x)} .
$$

First thing to note is that as we've been able to solve (2.49) for arbitrary $h(\tau)$, we might as well use $h(\tau)$ as given in (2.47). Thus, we have a boundary condition for $(2.53)^{0}$

$$
f(0)=1
$$

Second, the solution we need has a plus sign in (2.53): with the boundary condition (2.54), only this solution has a zero, which we find numerically to be at

$$
f\left(x_{\star}\right)=0 \quad \text { as } \quad x_{\star} \approx 0.55647 .
$$

Third, lacking the closed analytical solution for (2.53), we can solve it only as a power series for small $x$. For completeness, we present the first few terms in large $\tau$ (equivalent to the small $x$ ) expansion of the warp factors $\triangle_{i}(h(\tau))$ :

$$
\begin{aligned}
& \triangle_{1}(x)=1-\frac{3}{4} x-\frac{9}{32} x^{2}+O\left(x^{3}\right) \\
& \triangle_{2}(x)=1-\frac{3}{4} x-\frac{63}{160} x^{2}+O\left(x^{3}\right) .
\end{aligned}
$$

Given the solution to the warp factors $\triangle_{i}(\tau)$, we can go back and compute $c_{i}(\tau)$ (from (2.48) and (2.46)), the $\omega(\tau)$ (from (2.40), and solve (2.39) for the three-form. We will not attempt to solve these equations exactly, but

\footnotetext{
${ }^{8}$ Recall that the geometry should approach that of KT for large $\tau$.
} 
rather present the leading behavior of the metric at large $\tau$. To make contact with KT solution, we use a radial coordinate as in (2.18) and

$$
L^{4}=\frac{81}{2} g_{s} M^{2} \epsilon^{4}, \quad r_{0}^{4}=\frac{243 A}{2} .
$$

The metric is then given by

$$
d s_{10}^{2}=H(r)^{-1 / 2}\left[\triangle_{1}(r)^{2} d t^{2}-d \bar{x}^{2}\right]-H(r)^{1 / 2}\left[\frac{d r^{2}}{\triangle_{2}(r)^{2}}+r^{2} d s_{T^{1,1}}^{2}\right]
$$

with

$$
\begin{array}{r}
H(r)=\frac{L^{4}}{r^{4}} \ln \frac{r}{r_{s}}-\frac{L^{4} r_{0}^{4}}{8 r^{8}}+\cdots, \\
\triangle_{1}(r)=1-\frac{r_{0}^{4}}{2 r^{4}}+\cdots, \\
\triangle_{2}(r)=1-\frac{r_{0}^{4}}{2 r^{4}}+\cdots,
\end{array}
$$

where the dots indicate subdominant $1 / r$ and $\ln (r) / r$ corrections as $r \rightarrow \infty$. The event horizon $r_{\star}$ of the stationary black hole geometry occurs where $g^{r r}=0 \Leftrightarrow \triangle_{2}\left(r_{\star}\right)=0$. Numerically, we find

$$
\left(\frac{r_{0}}{r_{\star}}\right)^{4} \equiv \xi \approx 1.02427
$$

From (2.59), the black hole horizon will "cloak" a naked singularity in KT geometry if $H\left(r_{\star}\right)>0$. Assuming $L \gg r_{0}$, this condition translates into

$$
r_{0}>r_{s} \xi^{1 / 4} e^{\xi / 8} \approx 1.14343 r_{s}
$$

The fact that a black hole in KT geometry must be larger then of certain critical size (given by (2.61)) to hide the naked singularity, is the gravitational dual reflection that chiral symmetry is restored in the gauge theory at finite temperature. We return to this issue in the following section. 


\section{Thermodynamics of KT geometry}

In this section we compute the entropy of the black hole discussed in the previous section and compare it with the entropy of the dual gauge theory.

To compute the Hawking temperature of the black hole (2.31), we introduce the proper distance near the horizon:

$$
d \eta \approx c_{2}\left(\tau_{\star}\right) \triangle_{2}(\tau)^{-1} d \tau
$$

where $\tau_{\star}$ is the event horizon (2.60). Using (2.46), the latter can be rewritten as

$$
d \eta \approx c_{2}\left(\tau_{\star}\right) h\left(\tau_{\star}\right)^{-1} d \triangle_{1}
$$

or

$$
\eta(\tau) \approx c_{2}\left(\tau_{\star}\right) h\left(\tau_{\star}\right)^{-1} \triangle_{1}(\tau)
$$

Now, rescaling the time

$$
\tilde{t}=t \frac{c_{1}\left(\tau_{\star}\right) h\left(\tau_{\star}\right)}{c_{2}\left(\tau_{\star}\right)},
$$

the metric becomes

$$
d s_{10}^{2} \approx \eta^{2} d \tilde{t}^{2}-d \eta^{2}-c_{1}\left(\tau_{\star}\right)^{2} d \bar{x}^{2}-9 c_{2}\left(\tau_{\star}\right)^{2} d s_{T^{1,1}}^{2}
$$

From this form of the metric it is easy to see that if we Wick rotate $\tilde{t}$, we will avoid a conical singularity if we identify the Euclidean time $i \tilde{t}$ with period $2 \pi$. The periodicity in Euclidean time is identified as the inverse temperature. Tracing back to our original coordinate system, we identify the black hole temperature to be

$$
T_{H}=\frac{c_{1}\left(\tau_{\star}\right) h\left(\tau_{\star}\right)}{2 \pi c_{2}\left(\tau_{\star}\right)} .
$$

If $L \gg r_{0}$ (2.57) and (2.61) holds, we can estimate $T_{H}$ using asymptotics of the metric

$$
T_{H} \approx \frac{r_{\star}}{\pi L^{2}}\left(\frac{r_{0}}{r_{\star}}\right)^{4} \ln ^{-1 / 2} \frac{r_{\star}}{r_{s}}
$$

where

$$
r_{\star} \equiv \epsilon e^{\tau_{\star} / 3}
$$


For the following estimates we take $r_{\star} \approx r_{0}(2.60)$.

Next, we compute the Bekenstein-Hawking entropy of the geometry (2.31). We find the 8-dimensional area of the event horizon $\mathcal{A}_{8}$ of the black hole to be

$$
\mathcal{A}_{8}=c_{1}\left(\tau_{\star}\right)^{3}\left(3 c_{2}\left(\tau_{\star}\right)\right)^{5} V_{3} \omega_{T^{1,1}} \approx \frac{16 \pi^{3}}{27} V_{3} r_{0}^{3} L^{2} \ln ^{1 / 2} \frac{r_{0}}{r_{s}},
$$

where $\omega_{T^{1,1}}=16 \pi^{3} / 27$ is the area of the $T^{1,1}$ space (2.15), and $V_{3}$ is the 3 -dimensional volume. The entropy of the black hole is then

$$
S_{B H}=\frac{\mathcal{A}_{8}}{4 G_{N}} \approx \frac{1}{54 \pi^{3} g_{s}^{2} \alpha^{\prime 4}} V_{3} r_{0}^{3} L^{2} \ln ^{1 / 2} \frac{r_{0}}{r_{s}},
$$

or, using (3.68) and (2.57)

$$
S_{B H} \sim M^{4} V_{3} T_{H}^{3} \ln ^{2}\left(T_{H}\right) .
$$

From the ordinary statistical mechanics we know that the energy is $d E=$ $T d S$ and the free energy is given by $F=E-T S$. Thus, for our black hole we estimate

$$
\begin{aligned}
& E \sim \frac{3}{4} T S, \\
& F \sim-\frac{1}{4} T S,
\end{aligned}
$$

at high temperatures.

The first computation of entropy of a finite temperature gauge theory and its gravitational dual was reported in [35]. The analysis of [35] was limited to a free $\mathcal{N}=4 U(N)$ Yang-Mills theory, which gravitation dual is realized by a system of coincident near-extremal D3-branes. In the canonical ensemble, where temperature and volume are the independent quantities, the temperature of the YM theory should be set to the Hawking temperature in the supergravity. The Bekenstein-Hawking temperature of black 3-branes then agrees up to a factor of $4 / 3$ with the free field computation in the YM theory. We would like to compare the BH entropy of our black hole (3.72) with the entropy of the finite temperature Klebanov-Strassler duality cascade. As in [35], we will do only free field theory estimate. 
The entropy computation of KS duality cascade is simple. At temperature $T$, a typical energy of a weakly coupled bosonic mode is $\frac{3 \zeta(5 / 2)}{2 \zeta(3 / 2)} T \approx 0.770269 T$ while that of a fermionic mode is $\frac{3 \zeta(5 / 2)(4-\sqrt{2})}{2 \zeta(3 / 2)(4-2 \sqrt{2})} T \approx 1.70007 T$. KlebanovStrassler analysis [26] suggests that at energy $\mu \sim T$ the weakly coupled description of the duality cascade is in terms of $S U(N(T)+M) \times S U(N(T))$ gauge theory with two chiral superfields in $(\overline{N(T)+M}, N(T))$ representation and two chiral superfields in $(N(T)+M, \overline{N(T)})$ representation. $N(T)$ is given by (2.29). Altogether we have $N(T)^{2}+(N(T)+M)^{2}-2$ vectors (each one contributes two bosonic degrees of freedom), $4 N(T)(N(T)+M)$ complex scalars (each one contributes two bosonic degrees of freedom) and $N(T)^{2}+$ $(N(T)+M)^{2}-2+4 N(T)(N(T)+M)$ Weyl fermions (each one contributes two fermionic degrees of freedom). Recalling that in four dimensions a bosonic mode contributes $\frac{2 \pi^{2} V T^{3}}{45}$ to the entropy of the system (a fermionic mode contributes $7 / 8$ of the bosonic mode contribution), we find

$$
\begin{aligned}
S_{\text {gauge }}= & \pi^{2} V\left[N(T)^{2}+M N(T)+\frac{1}{6} M^{2}-\frac{1}{3}\right] T^{3} \\
& \sim M^{4} V T^{3} \ln (T)^{2},
\end{aligned}
$$

where in the last line we suppressed numerical coefficients. Once we identify the temperature of the gauge theory with the Hawking temperature of the dual gravitational background, the leading temperature dependence of (3.72) agrees with the leading temperature dependence of (3.74) up to a numerical coefficient. This agreement provides a nontrivial check on the duality cascade proposed in [26].

In the previous section we argued that a black hole in KT background must be large enough to hide the naked singularity. Using (3.67), the criterion (2.61) can be represented as

$$
T_{H}>T_{s} \equiv \frac{r_{s}}{\pi L^{2}} e^{\xi / 8} \sqrt{\frac{8}{\xi}}
$$

Eq. (3.75) suggests that the temperature of the Klebanov-Strassler gauge theory duality cascade should be larger than $T_{s}$ for chiral symmetry to be 
restored. It is tempting to speculate that $T_{s}$ is in fact the temperature of this phase transition. To settle this question, we would have to compare the free energies of the black hole solution reported here with the free energy of the black hole in the Klebanov-Strassler geometry [26], both having the same Hawking temperature.

\section{Discussion}

In this paper we found an exact black hole solution in the Klebanov-Tseytlin geometry [12]. We propose that this black hole realizes gravitation dual of the $\mathcal{N}=1$ gauge theory duality cascade recently discussed by Klebanov and Strassler [26] in the high temperature phase. More precisely, we expect the solution to be relevant at temperatures above chiral symmetry breaking temperature in the gauge theory. We showed that the entropy of KS duality cascade at high temperatures is reproduced by the Bekenstein-Hawking entropy of the gravitational dual up to a numerical factor. The entropy computation on the gauge theory side strongly relies on the RG "logarithmic running" of the rank $N$ of the $S U(N+M) \times S U(N)$ gauge theory, which is the main prediction of KS duality cascade. The agreement in the entropy computation thus provides a check for KS duality cascade.

There are several interesting future directions [36]. First of all, it will be extremely interesting to find the generalization of the above construction to the Klebanov-Strassler geometry of the deformed conifold [26]. This would allow us to study chiral symmetry breaking of the gauge theory within supergravity. KS geometry describes zero temperature confining vacuum of the gauge theory. As the temperature increases, we expect a thermal phase transition into deconfinig phase. Given a black hole solution in KS geometry, we would be able to study this phase transition and its relation to the chiral symmetry breaking.

Finally, in this paper we presented yet another example supporting the general idea of 28]. Here, a "good" naked singularity of 12 can be hidden behind the black hole horizon. Recently Pando Zayas and Tseytlin discussed 
the supergravity solution of 3-branes on resolved conifold [37. Much like in [12], the solution has a naked singularity in the IR. The singularity is of the repulson type. At this stage it is not known how this singularity is resolved, or, whether it can be resolved at all. The construction of a black hole solution in geometry [37] might shed some light on this problem.

\section{5 (Added) On the horizon singularity of the non-BPS background constructed in the pre- vious sections.}

The metric ansatz for the non-extremal generalization of the KT background is given by eq. (2.31) above, which for convenience we reproduce here

$$
d s_{10}^{2}=c_{1}(\tau)^{2}\left[\triangle_{1}(\tau)^{2} d t^{2}-d \bar{x}^{2}\right]-c_{2}(\tau)^{2}\left[\triangle_{2}(\tau)^{-2} d \tau^{2}+9 d s_{T^{1,1}}^{2}\right] .
$$

The main assumption in searching for the non-BPS generalizations of the KT geometry used here is that the three-form flux on $T^{1,1}$ continuous to be self-dual even for the non-extremal solution. This guarantees that the dilaton does not run. The type IIB equations of motion then determine (2.46), (2.47)

$$
\triangle_{2}(\tau)\left[\triangle_{1}(\tau)\right]^{\prime}=\frac{A}{c_{1}(\tau)^{4} c_{2}(\tau)^{4}} \equiv h(\tau)=\frac{81 A}{\epsilon^{4}} e^{-4 \tau / 3}
$$

where $A$ is the non-extremality parameter. The warp factor $\triangle_{1}(\tau)$ is given by

$$
\triangle_{1}(\tau)=\sqrt{f(h(\tau))}
$$

where $f$ satisfies $(2.53)$

$$
[f(x)]^{\prime}=\frac{3}{2} \frac{\left(30 x+2 \sqrt{135 x^{2}+400 f(x)}\right) f(x)}{9 x^{2}-40 f(x)} .
$$

with the boundary condition $f(0)=1$. All the other functions specifying

the metric and the fluxes are determined once $f(x)$ is known. In Section 2.3, this equation has not been solved analytically. 
Using (5.77) the metric (5.76) can be written as

$$
d s_{10}^{2}=c_{1}(\tau)^{2}\left[\triangle_{1}(\tau)^{2} d t^{2}-d \bar{x}^{2}\right]-c_{2}(\tau)^{2}\left[h(\tau)^{-2}\left(d \triangle_{1}\right)^{2}+9 d s_{T^{1,1}}^{2}\right]
$$

with the event horizon determined from equation?

$$
\triangle_{1}\left(\tau_{\star}\right)=0
$$

or, equivalently

$$
f\left(x_{\star}\right)=0
$$

Numerically, the solution to (5.82) was found for finite $x_{\star} \approx 0.55647$. From the asymptotic analysis of Section 2.3 it follows that for sufficiently small $A$ (with arbitrary nonzero $x_{\star}$ ) the metric warp factors $c_{i}$ are regular at $x_{\star}$. Then, the radial coordinate determined from

$$
d \eta=c_{2}(\tau) h(\tau)^{-1} d \triangle_{1}
$$

is well-defined near $x_{\star}$. We can see immediately from (5.80) that in this case the horizon is non-singular.

Above arguments crucially depend on the statement that $x_{\star}$ is finite. This is actually incorrect [38]. In [38] equation (5.79) has been solved exactly

$$
\begin{aligned}
x \equiv x(u) & =\frac{4 a}{3 b} e^{-4 a u} \sinh 4 b u \\
f(x) \equiv f(x(u)) & =e^{-8 a u}
\end{aligned}
$$

with $3 a^{2}=5 b^{2}$ and $a=243 A / 4>0$. From (5.84) we see that $x_{\star}=0$, and so $\eta$ of (5.83) is ill defined near horizon as $h\left(\tau_{\star}\right)=x_{\star}$. So, rather then cloaking the naked singularity of the KT geometry, the horizon of nonextremal solution presented here actually coincides with it [38]. Given the uniqueness of the non-extremal solution for the self-dual ansatz of the threeform fluxes, the latter must thus be relaxed for the gravitational background

\footnotetext{
${ }^{9}$ For regular (nonsingular) horizons condition $(5.81)$ is equivalent to $\triangle_{2}\left(\tau_{\star}\right)=0$, which was used in Section 2.3.
} 
dual to the high temperature phase of the cascading gauge theory of [26]. The system of second order equations in the radial variable describing nonextremal generalizations of the KT background whose solutions may have regular horizons is given in [38].

Finally, from the exact solution (5.84)

$$
\begin{aligned}
& x \sim e^{-4 u a(1-\sqrt{3 / 5})} \\
& f \sim e^{-8 u a}, \quad \text { as } \quad u \rightarrow \infty
\end{aligned}
$$

or $f(x) / x$ vanishes exponentially as $u \rightarrow \infty$. This exponential suppression is hard to capture in numerical analysis.

\section{Acknowledgements}

I would like to thank Oliver DeWolfe, Christopher Herzog, Gary Horowitz, Sunny Itzhaki, Igor Klebanov, Andrei Mikhailov, Aleksey Nudelman, Joe Polchinski, Leopoldo Pando Zayas and Arkady Tseytlin for illuminating discussions. I am especially grateful to Joe Polchinski for reading the manuscript. This work was supported in part by NSF grants PHY97-22022 and PHY9907949 . 


\section{References}

[1] J. Maldacena, "The large N limit of superconformal field theories and supergravity," Adv. Theor. Math. Phys. 2 (1998) 231, hep-th/9711200.

[2] E. Witten, "Anti-de Sitter space and holography," Adv. Theor. Math. Phys. 2 (1998) 253, hep-th/9802150.

[3] S. S. Gubser, I. R. Klebanov and A. M. Polyakov, "Gauge theory correlators from non-critical string theory," Phys. Lett. B428 (1998) 105, hep-th/9802109.

[4] O. Aharony, S. S. Gubser, J. Maldacena, H. Ooguri and Y. Oz, "Large N Field Theories, String Theory and Gravity," Phys.Rept. 323, 183 (2000), hep-th/9905111.

[5] M. Douglas and G. Moore, "D-branes, Quivers, and ALE Instantons," hep-th/9603167.

[6] A. Kehagias, "New Type IIB Vacua and their F-Theory Interpretation," Phys. Lett. B435 (1998) 337, hep-th/9805131.

[7] I. R. Klebanov and E. Witten, "Superconformal Field Theory on Threebranes at a Calabi-Yau Singularity," Nucl. Phys. B536 (1998) 199, hepth/9807080.

[8] D. R. Morrison and M. R. Plesser, "Non-Spherical Horizons, I," Adv. Theor. Math. Phys. 3 (1999) 1, hep-th/9810201.

[9] J. Polchinski, "Tensors from K3 Orientifolds," Phys.Rev. D55 (1997) 6423, hep-th/9606165.

[10] I. R. Klebanov and N. Nekrasov, "Gravity Duals of Fractional Branes and Logarithmic RG Flow," Nucl.Phys. B574 (2000) 263, hepth/9911096. 
[11] K. Oh and R. Tatar, "Renormalization Group Flows on D3 branes at an Orbifolded Conifold," JHEP 0005 (2000) 030, hep-th/0003183.

[12] I. R. Klebanov and A. A. Tseytlin, "Gravity Duals of Supersymmetric SU(N) x SU(N+M) Gauge Theories," Nucl.Phys. B578 (2000) 123, hep-th/0002159.

[13] C. V. Johnson, A. W. Peet and J. Polchinski, "Gauge Theory and the Excision of Repulson Singularities," Phys.Rev. D61 (2000) 086001, hepth/9911161.

[14] L. Jarv and C. V. Johnson, "Orientifolds, M-Theory, and the ABCD's of the Enhancon," hep-th/0002244.

[15] K. Pilch and N. P. Warner, "N=2 Supersymmetric RG Flows and the IIB Dilaton," hep-th/0004063.

[16] A. Buchel, A. W. Peet and J. Polchinski, "Gauge Dual and Noncommutative Extension of an N=2 Supergravity Solution," hep-th/0008076.

[17] N. Evans, C. V. Johnson and M. Petrini, "The Enhancon and N=2 Gauge Theory/Gravity RG Flows," JHEP 0010 (2000) 022, hepth/0008081.

[18] M. Bertolini, P. Di Vecchia, M. Frau, A. Lerda, R. Marotta and I. Pesando, "Fractional D-branes and their gauge duals," hep-th/0011077.

[19] J. Polchinski and M. J. Strassler, "The String Dual of a Confining FourDimensional Gauge Theory," hep-th/0003136.

[20] I. Bena, "The M-theory dual of a 3 dimensional theory with reduced supersymmetry," hep-th/0004142.

[21] O. Aharony and A. Rajaraman, "String Theory Duals for Massdeformed $\mathrm{SO}(\mathrm{N})$ and $\mathrm{USp}(2 \mathrm{~N}) \mathrm{N}=4$ SYM Theories," Phys.Rev. $D 62$ (2000) 106002, hep-th/0004151. 
[22] I. Bena and A. Nudelman, "Warping and vacua of $(S) Y M_{3+1}$," Phys.Rev. D62 (2000) 086008, hep-th/0005163.

[23] I. Bena and A. Nudelman, "Exotic polarizations of D2 branes and oblique vacua of (S) $\mathrm{YM}_{2+1}$," hep-th/0006102.

[24] M. Cvetic, H. Lu and C. N. Pope, "Brane Resolution Through Transgression," hep-th/0011023.

[25] R. C. Myers, "Dielectric-Branes," JHEP 9912 (1999) 022, hepth/9910053.

[26] I. Klebanov and M. Strassler, "Supergravity and a Confining Gauge Theory: Duality Cascades and $\chi$ SB-Resolution of Naked Singularities," JHEP 0008 (2000) 052, hep-th/0007191.

[27] E. Witten, "Anti-de Sitter Space, Thermal Phase Transition, And Confinement In Gauge Theories," Adv.Theor.Math.Phys.2 (1998) 505, hepth/9803131.

[28] S. S. Gubser, "Curvature Singularities: the Good, the Bad, and the Naked," hep-th/0002160.

[29] D. Z. Freedman and J. A. Minahan, "Finite Temperature Effects in the Supergravity Dual of the N=1* Gauge Theory," hep-th/0007250.

[30] J. H. Schwarz, "Covariant Field Equations of Chiral $N=2 D=10$ Supergravity," Nucl.Phys. B226 (1983) 269.

[31] P. Candelas and X. C. de la Ossa, "Comments On Conifolds," Nucl.Phys. B342 (1990) 246.

[32] R. Minasian and D. Tsimpis, "On the geometry of non-trivially embedded branes," Nucl.Phys.B572 (2000) 499, hep-th/9911042.

[33] N. Seiberg, "Electric-Magnetic Duality in Supersymmetric Non-Abelian Gauge Theories," Nucl.Phys. B435 (1995) 129, hep-th/9411149. 
[34] A. W. Peet, "TASI lectures on black holes in string theory," hepth/0008241.

[35] S. S. Gubser, I. R. Klebanov and A. W. Peet, "Entropy and Temperature of Black 3-Branes," Phys.Rev.D54 (1996) 3915, hep-th/9602135.

[36] Work in progress.

[37] L. A. Pando Zayas and A. A. Tseytlin, "3-branes on resolved conifold," hep-th/0010088.

[38] A. Buchel, C. P. Herzog, I. R. Klebanov, L. Pando Zayas and A. A.Tseytlin, "Non-Extremal Generalizations of D3-Brane Background with Logarithmically Varying Flux", hep-th/0102105. 